ESJ Humanities

\title{
Positive Body Image of Female Students: A Predictive Study of the Integrative Body Image Model
}

\author{
Egle Urvelyte \\ Aidas Perminas \\ Vytauto Didžiojo Universitetas, Lithuania
}

Doi:10.19044/esj.2021.v17n24p1

Submitted: 20 May 2021

Accepted: 05 July 2021

Published: 31 July 2021
Copyright 2021 Author(s)

Under Creative Commons BY-NC-ND

4.0 OPEN ACCESS

Cite As:

Urvelyte E. \& Perminas A. (2021). Positive Body Image of Female Students: A Predictive Study of the Integrative Body Image Model. European Scientific Journal, ESJ, 17(24), 1. https://doi.org/10.19044/esj.2021.v17n24p1

\begin{abstract}
This paper focuses on testing hypothesized psychosocial predictors factors (general unconditional acceptance, body acceptance by others, body function appreciation) for positive body image among 812 female students aged between 18 and 35 years. Path analysis procedures contained in the Mplus Version 7 program were used to evaluate the positive body image model. The positive body image model indicated that greater perceived body acceptance by others was linked to greater body function appreciation which in turn was linked to higher positive body image. Perceived body image acceptance by others was directly related to higher positive body image. General unconditional acceptance did not lead to female students greater body function appreciation. The findings revealed some important positive body image predicting factors, and these results can be used to improve positive body image interventions.
\end{abstract}

Keywords: Female students, positive body image model

\section{Introduction}

Body image is accepted to be a multidimensional and complex construct (Pruzinsky \& Cash, 2002). For a long time, majority of studies related to body image focused on body image dissatisfaction and its risk factors. However, a more recent step in these studies has become the direction of positive psychology (Seligman \& Csikszentmihalyi, 2000). Researchers 
have tried to understand the causes of negative body image, but it is also important to understand and promote a positive side of body image. Positive body image has often been understood as the absence of negative body image according to proponents of positive body image. However, it is not only in reverse to dissatisfaction with body image but it has unique aspects to understand (Tylka, 2011). Positive body image includes aspects such as positive opinion of your body (regardless of its appearance); acceptance of the body (regardless of body weight and shapes); respect for one's body (taking care of it, following a healthy lifestyle and responding to the needs of the body); and rejection of stereotypes in the media (to protect one's own body). These aspects are usually included in instruments for assessing positive body image (Avalos, Tylka \& Wood-Barcalow, 2005).

People with positive body image tend to dismiss beauty stereotypes in the media and perceive beauty as a much broader construct, believing that each person and his body is unique and beautiful (Tylka, 2013).

Studies have shown that positive body image is multifaceted, holistic, formed during interventions, and acts as a protective factor (Tylka \& WoodBaecalow, 2015). Positive body image can be stable and variable. If a positive image of the body is seen as a personality trait, then it is believed that it can be stable and immutable. Nevertheless, there is growing evidence in scientific studies that positive body image is not just a stable personality trait. Albertson, Neff, and Dill-Shackleford (2014) applied meditation practices to women who were concerned about their body image during their study. The intervention helped to increase the positive image of the female body, and the effect persisted after 3 months of re-measurement. This indicates that the positive image of the body can be changed during interventions, but at the same time achieved effect remains to be quite stable. A positive body image is a separate construct from body image dissatisfaction. In other words, it is not only the opposite of a negative body image. Positive body image is not in the same continuum as negative body image, neither can positive body image be seen only as a low level of body image dissatisfaction (Tylka, 2011, 2013).

The fact that positive body image and negative body image are not a mirror image of each other is also justified by research results. Tiggeman and McCourt (2013) noted in their results that women can experience a certain level of dissatisfaction with body image at the same time, but also appreciate and feel respect for their body. A study of older athlete women (over 50 years old) found that while they invest in their appearance because they are somewhat dissatisfied with their body, they also tend to appreciate the functionality of their body (Bailey, Cline \& Gammage, 2016). Furthermore, a study of teenage girls revealed that teenagers distinguish characteristics they would like to change in their appearance, but they also have positive feelings about their body (Pope, Corona \& Belgrave, 2014). 
Studies have shown that positive body image is positively related to both physical health and psychological well-being (Avalos et al., 2005). Studies have also indicated that positive body image is positively associated with intuitive eating in groups of adult women, adolescents, and athlete students (Hahn Oh, Wiseman, Hendrickson, Phillips \& Hayden, 2012; Tylka \& Kroon Van Diest, 2013; Andrew, Tiggemann \& Clark, 2015). It is also associated with a sense of compassion in the group of female students (Homan \& Tylka, 2015; Wasylkiw, MacKinnon \& MacLellan, 2012), greater students life satisfaction (Tylka \& Kroon Van Diest, 2013), and greater sexual satisfaction in adult women (Satinsky, Reece, Dennis, Sanders \& Bardzell, 2012).

Despite the increasing interest in positive body image, only a few studies have examined predictors of positive body image (Andrew et al., 2016; Avalos et al., 2005; Iannantuono \& Tylka, 2012; Augustus-Horvath \& Tylka, 2011). However, studies of the factors predicting positive body image are important from both a theoretical and a practical point of view so as to develop interventions that will enhance positive body image.

This study focused on developing an integrated model of positive body image (Figure 1). Some mediation and prognostic factors were also taken from the acceptance model of intuitive eating (Avalos et al., 2005).

The first two psychological predictors, i.e., perceived body acceptance by others and perceived general unconditional acceptance was investigated. In this model, perceived body acceptance by others and general unconditional acceptance is conceptualised to lead to increased body functionality. In addition, it was predicted that body function would explain or mediate the relationship between body acceptance by others, unconditional acceptance, and positive body image.

Furthermore, research has shown that women who perceive low level of general acceptance and body acceptance from others feel more pressure to lose weight and are unhappy with their body image (Tylka, Subich, 2002). Women who focus on how their bodies function rather than their external appearance are believed to be more likely to appreciate their bodies (Tylka, 2006). 


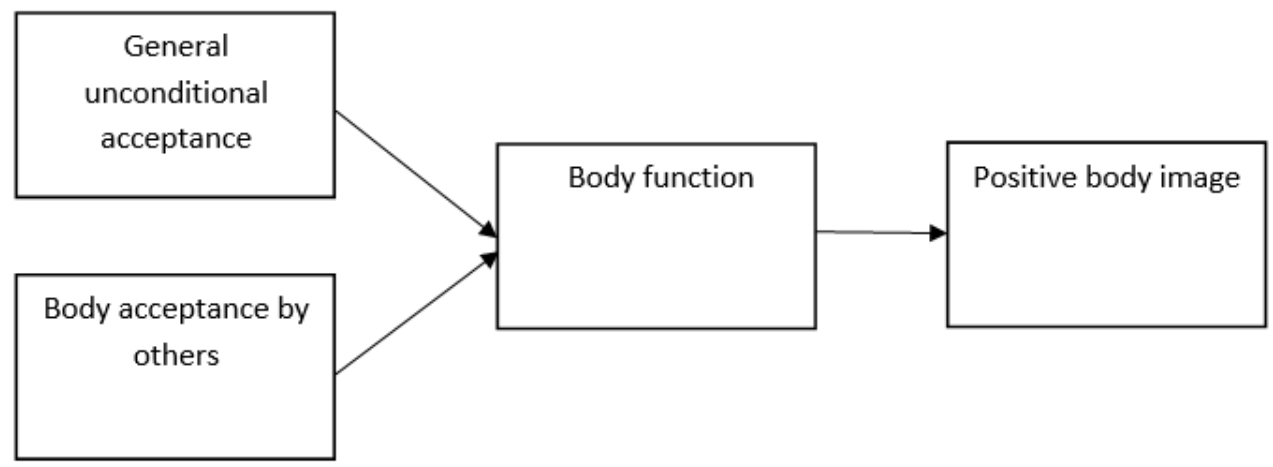

Figure 1. The hypothesized model of positive body image

In this study, the following hypotheses were examined:

Hypothesis 1: Perceived general unconditional acceptance and body image acceptance from others predicts female students' emphasis on body function; Hypothesis 2: Body function predicts positive body image;

Hypothesis 3: The link of perceived general unconditional acceptance and perceived body image from others to positive body image is fully mediated by body function.

\section{Research Methods and Material \\ 2.1 Procedure}

The survey was conducted over the internet and required participation in a study on body image from respondents who were studying in three universities in Kaunas city. Those who agreed to participate provided written informed consent and completed an anonymous online version of the questionnaire. Participation was voluntary and respondents did not receive any remuneration for participation.

\subsection{Participants}

A sample of 812 female students from three universities in Kaunas city, Lithuania, participated in this study with an age range of 18 to 35 years $(\mathrm{M}=21.47, \mathrm{SD}=2.48)$. The mean self-reported body mass index (BMI) was $22.34(\mathrm{SD}=3.75)$, which falls within the 'normal range' for adult women (World Health Organization, 2015).

\subsection{Measures}

Based on the validation of the original 86-item Barrett-Lennard Relationship Inventory (BLRI; Barrett-Lennard, 1962), 8-item version was used to measure students' perceived general unconditional support from a significant other. Participants were instructed to think about the most important and influential person in their life and to answer the items (e.g., 
"She/he nearly always knows exactly what I mean") on a 6-point scale ranging from 1 (very false) to 6 (very true) with that person in mind.

The Body Acceptance by Others Scale (BAOS) (Avalos et al., 2005) was used to measure students perceived body image acceptance by others. Participants rated the BAOS items (e.g., "I've felt acceptance from my friends regarding my body shape and/or weight") on a 5-point scale ranging from 1 (never) to 5 (always).

Body function was assessed by The Functionality Appreciation Scale (FAS) (Alleva, Tylka Kroon Van Diest, 2017). Participants rated the FAS items (e.g., "I respect my body for the functions that it performs") on a 5-point scale ranging from 1 (strongly agree) to 5 (strongly disagree).

To assess positive body image, the 10-item Body Appreciation Scale2 was used (Final Version) (BAS-2; Tylka, Wood-Barcalow, 2015). The BAS's-2 items (e.g., "I respect my body") are rated on a 5-point scale ranging from 1 (never) to 5 (always) and averaged.

\section{Results}

Path analysis procedures contained in the Mplus Version 7 program were used to evaluate the positive body image model presented in Figure 1. Since all measures were continuous and univariate skewness and kurtoses in the Mplus output suggest non-normality, the maximum likelihood (MLR) parameter estimates with standard errors and a chi-square test statistic was used. Adequacy of fit, which was recommended by Hu and Bentler (1999), was determined by four indices, namely: the comparative fit index (CFI), the Tucker-Lewis Index (TLI), the standardized rootmean-square residual (SRMR), and the root-mean-square error of approximation (RMSEA).

The acceptance model provided an excellent fit to the data $(\mathrm{CFI}=1.00$, $\mathrm{TLI}=1.01, \mathrm{SRMR}=.0002$, RMSEA<.001). All paths were significant except for the path from general unconditional acceptance to body function $(\beta=(-$ $.05), \mathrm{p}=.132)$;

The path coefficients for this model are presented in Figure 2 and Table 1. Approximately, $14 \%$ of the variance in body function was explained by body acceptance by others and $56 \%$ of the variance in positive body image was explained by body function and body acceptance by others. 


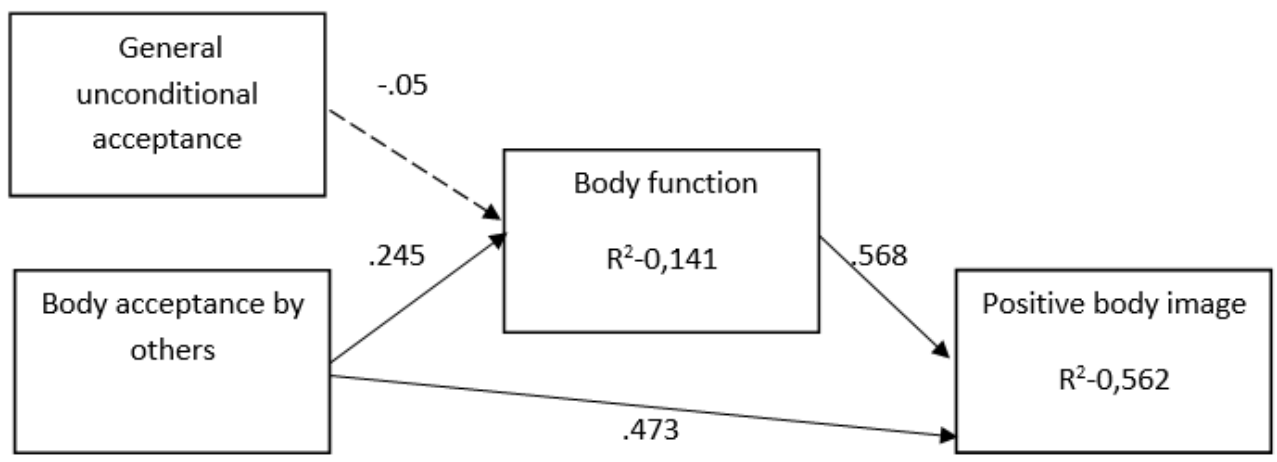

Figure 2. Paths coefficients for the positive body image model Note: $\longrightarrow$ significant path; $\longrightarrow$ insignificant path.

Furthermore, the body function was tested to observe whether it fully mediated the positive body image. 5,000 bootstrap samples were created from the data set $(\mathrm{N}=812)$ by random sampling with replacement. Within the structural model, Mplus was specified to estimate indirect effect for the body acceptance by others, body function, and positive body image. Results indicated that the 95\% CIs for the indirect effects did not include zero, indicating that indirect path was statistically significant. When body function was examined as a mediator between body acceptance by others and positive body image, the standardized indirect effect was $\beta=.087$, the mean indirect (unstandardized) effect was 0.246 , the standard error of the mean indirect effect was .069 , and the $95 \%$ CI for the mean indirect effect was .040 (lower limit) and .134 (upper limit) (Table 1).

Table 1. Positive body image model paths results

\begin{tabular}{|c|c|c|c|c|c|c|}
\hline & $\beta$ & St. $\beta$ & SD & $\mathbf{p}$ & $95 \%$ & \\
\hline $\begin{array}{l}\text { Body acceptance by others } \\
\text { body function } \longrightarrow\end{array}$ & .245 & .362 & .024 & $<.001$ & $\begin{array}{l}.198 \\
.293\end{array}$ & - \\
\hline $\begin{array}{l}\text { General acceptance } \longrightarrow \text { body } \\
\text { function }\end{array}$ & -.050 & -.048 & .033 & .132 & $\begin{array}{l}-.116 \\
.014\end{array}$ & - \\
\hline $\begin{array}{l}\text { Body acceptance from others } \\
\text { positive body image } \longrightarrow\end{array}$ & .473 & .436 & .034 & $<.001$ & $\begin{array}{l}.407 \\
.539\end{array}$ & - \\
\hline $\begin{array}{l}\text { Body function } \longrightarrow \text { positive body } \\
\text { image }\end{array}$ & .568 & .354 & .061 & $<.001$ & $\begin{array}{l}.454 \\
.697 \\
\end{array}$ & - \\
\hline $\begin{array}{l}\text { Body acceptance from others } \longrightarrow \\
\text { body function } \longrightarrow \text { positive body }\end{array}$ & .246 & .087 & .069 & $<.001$ & $\begin{array}{l}.040 \\
.134\end{array}$ & - \\
\hline
\end{tabular}

image

Note: $\mathrm{N}=812$. $\mathrm{B}$ - regression coefficient; St. $\beta$ - standardized regression coefficient; SD standard deviation; 95\% CI - Bias-corrected confidence intervals.

\section{Discussion and Conclusions}

This study examined factors that predicted positive body image in female students. Specifically, the study examined general unconditional 
acceptance, body image acceptance by others, and body function as predictors of positive body image.

The positive body image model indicated that greater body acceptance by others was linked to greater body function appreciation which in turn was linked to higher positive body image. Also, body image acceptance by others was directly related to higher positive body image. However, general unconditional acceptance did not lead to female students greater body function appreciation.

In this case, the results supported that instead of general unconditional acceptance, specific type of acceptance (body acceptance by others) is associated with students' greater body function appreciation. Thus, body image acceptance by others predicted students' positive body image. According to Stice, Nemeroff, and Shaw (1996), perceived pressure to be thin is associated with higher body image dissatisfaction. This research together with the findings in this study revealed that to improve women body image, it is important to accept their body rather than increase pressure to loose weight. In agreements with other research (Tylka, 2006; Avalos et al., 2005), positive body image model revealed that when women appreciate their body functionality more than their appearance, they are more likely to have positive body image.

The findings of this study further revealed some important positive body image predicting factors such as body acceptance by others and body function appreciation. Also, these results can be used to improve positive body image interventions. This would involve teaching women to appreciate their body functionality.

However, this study faced some limitations. First of all, general unconditional acceptance was measured by only thinking about one important person in their life when growing up. Maybe for some participants, it was not only one important person which gave them acceptance. Another limitation in the analysis that was not included is the BMI (body mass index). It could be that women with higher BMI may have received less body image acceptance from others.

\section{References:}

1. Amy Iannantuono, C. \& Tracy Tylka, L. (2012). Interpersonal and intrapersonal links to body appreciation in college women: An exploratory model. , 9(2), 227-235. doi:10.1016/j.bodyim.2012.01.004

2. Andrew, R., Tiggemann, M., \& Clark, L. (2015). The protective role of body appreciation against media-induced body dissatisfaction. Body Image, 15, 98-104. https://doi.org/10.1016/j.bodyim.2015.07.005 
3. Augustus-Horvath Casey, L. \& Tylka Tracy, L. (2011). The acceptance model of intuitive eating: A comparison of women in emerging adulthood, early adulthood, and middle adulthood.. Journal of Counseling Psychology, 58(1), 110-125. doi:10.1037/a0022129

4. Avalos, L., Tylka, L., \& Wood-Barcalow, N. (2005). The body Appreciation Scale: Development and psychometric evaluation., 2 (3), 285-297. doi:10.1016/j.bodyim.2005.06.002

5. Bailey, K. A., Gammage, K. L., \& Van Ingen, C. (2017). How do you define body image? Exploring conceptual gaps in understandings of body image at an exercise facility. Body Image, 23, 69-79. https://doi.org/10.1016/j.bodyim.2017.08.003

6. Baker, C. \& Wertheim, E. H. (2003). Body Image: A Handbook of Theory, Research, and Clinical Practice, edited by Thomas F. Cash and Thomas Pruzinsky, New York: Guilford Press, 2002, 530 pages, \$60.00. Eating Disorders, 11(3), 247-248.

https://doi.org/10.1080/10640260390218738

7. Homan, K. J. \& Tylka, T. L. (2015). Self-compassion moderates body comparison and appearance self-worth's inverse relationships with body appreciation. Body Image, 15, 1-7. https://doi.org/10.1016/j.bodyim.2015.04.007

8. Louise Wasylkiw, Anna MacKinnon, L., \& Aleka MacLellan, M. (2012). Exploring the link between self-compassion and body image in university women. , 9(2), 236-245. doi:10.1016/j.bodyim.2012.01.007

9. Oh, K. H., Wiseman, M. C., Hendrickson, J., Phillips, J. C., \& Hayden, E. W. (2012). Testing the Acceptance Model of Intuitive Eating With College Women Athletes. Psychology of Women Quarterly, 36(1), 8898. https://doi.org/10.1177/0361684311433282

10. Pope, M., Corona, R., \& Belgrave, F. Z. (2014). Nobody's perfect: A qualitative examination of African American maternal caregivers' and their adolescent girls' perceptions of body image. Body Image, 11(3), 307-317. https://doi.org/10.1016/j.bodyim.2014.04.005

11. Seligman Martin, E. P. \& Csikszentmihalyi Mihaly (2000). Positive psychology: An introduction.. , 55(1), 5-14.

doi:10.1037/0003-066x.55.1.5

12. Sonya Satinsky, Michael Reece, Barbara Dennis, Stephanie Sanders, $\&$ Shaowen Bardzell (2012). An assessment of body appreciation and its relationship to sexual function in women. 9(1), 137-144. doi:10.1016/j.bodyim.2011.09.007

13. Tiggemann, M. \& McCourt, A. (2013). Body appreciation in adult women: Relationships with age and body satisfaction. Body Image, 10(4), 624-627. https://doi.org/10.1016/j.bodyim.2013.07.003 
14. Tylka, T. L. (2011). Positive psychology perspectives on body image. In T. F. Cash \& L. Smolak (Eds.), Body image: A handbook of science, practice, and prevention (p. 56-64). The Guilford Press.

15. Tylka, T. L. \& Kroon Van Diest, A. M. (2013). The Intuitive Eating Scale-2: Item refinement and psychometric evaluation with college women and men. Journal of Counseling Psychology, 60(1), 137-153. https://doi.org/10.1037/a0030893

16. Tylka, T. L. \& Subich, L. M. (2002). A preliminary investigation of the eating disorder continuum with men. Journal of Counseling Psychology, 49, 273-279

17. Tylka, T.L. (2013). Evidence for body appreciation scale's measurement equivalence/invariance between U.S. college women and men. Body Image, 10, 415-418.

doi:10.1016/j.bodyim.2013.02.006 